\title{
On the jet spectrum in nucleus-nucleus interactions
}

\author{
M.A.Braun ${ }^{1}$, E.G.Ferreiro ${ }^{2}$, C.Pajares ${ }^{2}$ and D.Treleani ${ }^{3}$ \\ ${ }^{1}$ Department of High Energy Physics, University of St. Petersburg, \\ 198904 St. Petersburg, Russia \\ ${ }^{2}$ Departamento de Fisica de Particulas, Unicersidade de Santiago de Compostela \\ 15706 Santiago de Compostela, Spain \\ ${ }^{3}$ Dipartimento di Fisica Teorica dell'Università di Trieste and INFN, Sezione di Trieste \\ Strada Costiera 11, Miramare-Grignano, I-34014, Trieste, Italy
}

\begin{abstract}
We derive the inclusive transverse spectrum of minijets in nuclear collisions at very high energies, by assuming that the nuclear $S$-matrix factorizes as a product elastic $S$-matrices of elementary partonic collisions. Interference effects and, in particular, the contribution of loop diagrams are fully taken into account in the derivation of the spectrum, which is shown to coincide with the result already obtained by superposing the elementary interactions incoherently. A quantitative analysis confirms that the deformation induced by multiple collsions is a large effect at RHIC and LHC energies, for transverse momenta $\sim 20 \mathrm{GeV}$.
\end{abstract}

\section{Introduction}

The growing experimental activity in heavy-ion collision has stimulated a renewed interest on the $p_{T}$ distributions of secondaries in AB collisions. From the theoretical point of view a special attractiveness presents the production of jets, which, in principle, admits a study in the framework of perturbative QCD. In fact theoretical predictions for the inclusive jet production in $p \bar{p}$ collisions at $1800 \mathrm{GeV}$ agree quite well with the experimental data for jet transverse energies in the region $50 \div 250 \mathrm{GeV} / \mathrm{c}[1]$. In the region of lower jet transverse momenta, the agreement is not as good and phenomenogical parameters need to be introduced [2] [3]. In nuclear collisions the situation becomes obviously much more complicated. There are several mechanisms which influence the form of the jet spectrum and make it different from the case of $p \bar{p}$. In the nucleus the partonic distributions are changed, the produced jets may loose some energy while passing through the thick nuclear matter, jets may accumulate more transverse momentum by hard rescatterings. All these different phenomena need to be disentangled to gain a comprehensive understanding of the production mechanism.

In the present paper we focus on the effects of rescattering on the transverse spectrum of minijets in nucleus-nucleus collisions. The problem was studied in the past assuming that the total inelastic hard cross-section can be obtained from the forward scattering amplitude by substituting the partonic (elastic) cross-sections for the forward parton-parton scattering amplitude[1] [5], similarly to the 
analogous case, where the inelastic nucleus-nucleus cross section is expressed in terms of the nucleon-

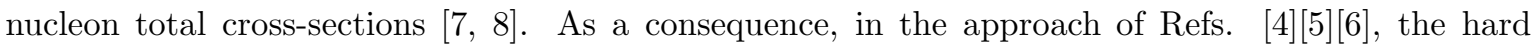
nucleus-nucleus cross section is expressed as an incoherent superposition of interactions between the partons of the nuclear structures. Possible coherence effects are not included and, in particular, the well known problem of loops, arising in the Glauber approach to nucleus-nucleus collisions[9], is completely disregarded.

The purpose of the present paper is to take interferences into account and include all loops in the evaluation of rescatterings in the inclusive spectrum of minijet production in nucleus-nucleus collisions. In our approach each colliding nucleus will be assumed to consist of a variable number of partons, distributed both in impact parameter and rapidity, while the nuclear $S$-matrix will be assumed to factorize as a product of parton-parton $S$-matrices. The inclusive cross-section for minijet production will be derived without approximations, apart from the usual disentangling of the longitudinal and transverse degrees of freedom and, as we will show, the result will turn out to be identical to the expression already obtained in 四河递] under the incoherent no-loop approximation.

As an application, in the second part of the paper we study the $p_{T}$ distributions in central $\mathrm{Pb}$ $\mathrm{Pb}$ collisions at nucleon-nucleon c.m energies of 200 and $6000 \mathrm{GeV}$, corresponding to the regimes at RHIC and LHC. Since our aim is not to fit the existing data but rather to see the influence of rescatterings, we compare our results with the naive picture where each projectile parton is allowed to interact with a single target parton only, weighted with the partonic distributions of both nuclei, which are taken to be identical to those of the proton. Rescattering effects are obviously maximal at relatively small transverse momenta, where the partonic cross-section is relatively large. For this reason we concentrate our attention on the production of minijets with transverse momenta up to 10 $\div 20 \mathrm{GeV} /$ c. To make our picture compatible with the existing experimental data in $p \bar{p}$ collisions at this momenta, we shall use an efective partonic cross-sections taken from the lowest order pertubative QCD and corrected by phenomenological parameters as in [2] [3].

\section{Glauber theory for hard parton scattering}

We start by introducing the expression for the hard scattering amplitude in terms of partonic interactions by following the philosophy of the Glauber approach to nucleus-nucleus collisions. Hence we assume 1) conservation of partonic longitudinal momenta and impact parameters and 2) factorization of the $S$ matrix into the product of elementary partonic $S$ matrices.

The nucleus state $|A\rangle$ can be represented by a superposition of states with a different number $n$ of partons, each characterized by its scaling variable $x$ and impact parameter $b$. For brevity we denote $z=\{x, b\}$. We also consider only one partonic flavour, although the generalization to different flavours $f$ is trivial. With these notations one has

$$
|A\rangle=\sum_{n} \int \prod_{i=1}^{n} d^{3} z_{i} \Psi_{A, n}\left(z_{i}\right)\left|n, z_{i}\right\rangle
$$

In the following we shall denote the phase space of the $n$-parton configuration in nucleus $\mathrm{A}$ as

$$
d \tau_{A}(n)=\prod_{i=1}^{n} d^{3} z_{i}
$$

For the nucleus A - nucleus B collision the $S$ matrix has the form

$$
\begin{gathered}
\left\langle A^{\prime} B^{\prime}|S| A B\right\rangle=\sum_{n, l, n^{\prime}, l^{\prime}} \int d \tau_{A}(n) d \tau_{A}\left(n^{\prime}\right) d \tau_{B}(l) d \tau_{B}\left(l^{\prime}\right) \\
\Psi_{A^{\prime}, n^{\prime}}^{*}\left(z_{i}^{\prime}\right) \Psi_{B^{\prime}, l^{\prime}}^{*}\left(u_{j}^{\prime}\right) \Psi_{A, n}\left(z_{i}\right) \Psi_{B, l}\left(u_{j}\right)\left\langle n^{\prime}, z_{i}^{\prime} ; l^{\prime}, u_{j}^{\prime}|S| n, z_{i} ; l, u_{j}\right\rangle
\end{gathered}
$$


Here $u_{j}$ are the partonic variables of the nucleus $\mathrm{B}$, being $l$ the number of partons in a given configuration. As stated, our first assumption is that the $S$ matrix is diagonal in the basis $\left\{n, z_{i} ; l, u_{j}\right\}$ :

$$
\left\langle n^{\prime}, z_{i}^{\prime} ; l^{\prime}, u_{j}^{\prime}|S| n, z_{i} ; l, u_{j}\right\rangle=\delta_{n n^{\prime}} \delta_{l l^{\prime}} \prod_{i=1}^{n} \delta^{3}\left(z_{i}-z_{i}^{\prime}\right) \prod_{j=1}^{l} \delta^{3}\left(u_{i}-u_{i}^{\prime}\right) S_{n l}\left(z_{1}, \ldots z_{n} \mid u_{1}, \ldots u_{l}\right)
$$

Physically this assumption corresponds taking into account binary collisions only, therefore conserving the number of partons in the hard process, which may be justified arguing that in a hard interaction parton production is damped by an extra power of the strong coupling constant. We also assume that neither the longitudinal momentum nor the impact parameter are changed in a hard collision, which are basic assumptions in the Glauber approach. The range in $x$ and $p_{\perp}$ for which the treatment can be applied is therefore restricted to the region where longidudinal momenta are much higher as compared to the transferred momenta. Under assumption (4) the $S$ matrix simplifies to

$$
\left\langle A^{\prime} B^{\prime}|S| A B\right\rangle=\sum_{n, l} \int d \tau_{A}(n) d \tau_{B}(l)\left|\Psi_{A, n}\left(z_{i}\right)\right|^{2}\left|\Psi_{B, l}\left(u_{j}\right)\right|^{2} S_{n l}\left(z_{1}, \ldots z_{n} \mid u_{1}, \ldots u_{l}\right)
$$

A second assumption intrinsic in the Glauber approach is that of factorization of the $S$ matrix in (5) into a product of elementary $S$ matrices for parton-parton scatterings

$$
S_{n l}\left(z_{1}, \ldots z_{n} \mid u_{1}, \ldots u_{l}\right)=\prod_{i=1}^{n} \prod_{j=1}^{l} s_{i j}
$$

where $s_{i j}$ is the $S$ matrix for the collision of parton $i$ from nucleus A with parton $j$ from nucleus B:

$$
s_{i j}=1+i a\left(z_{i}, u_{j}\right) \equiv 1+i a_{i j}
$$

$a$ being the parton-parton scattering amplitude. Since we assume that no parton production takes place, $s_{i j}$ corresponds to purely elastic scatterings (or to binary reactions in the multi-flavour case). Unitarity is therefore satisfied in its simplest form

$$
\left(s_{i j}\right)^{*} s_{i j}=1
$$

(in the multi-flavour case this is changed into a matrix condition in the flavour space)

To complete the picture we have to introduce the normalization condition for the nuclear wave function, which reads

$$
\sum_{n} \int d \tau_{A}(n)\left|\Psi_{A, n}\left(z_{i}\right)\right|^{2}=1
$$

As one observes the normalization condition does not limit the parton population of a given nuclear configuration, represented by a given term in the sum, it only restricts the total probability of the different configurations to be unity. The cross-section introduced in [4] - [6] corresponds to choosing the distribution in the number of partons to be Poissonian:

$$
w_{n}=\int d \tau(n)\left|\Psi_{A, n}\left(z_{i}\right)\right|^{2}=e^{-\langle n\rangle} \frac{\langle n\rangle^{n}}{n !}
$$

If one additionally assumes that the the nuclear partonic wave function factorizes as

$$
\left|\Psi_{A, n}\left(z_{i}\right)\right|^{2}=c_{n} \prod_{i=1}^{n} \Gamma\left(z_{i}\right)
$$

one immediately gets

$$
c_{n}=\frac{1}{n !} e^{-\langle n\rangle}, \quad\langle n\rangle=\int d^{3} z \Gamma(z)
$$


With the forward AB scattering amplitude defined by (5)- (7), we can proceed to determine the relevant cross-sections. The total cross-section is of course obtained from the imaginary part of the forward $\langle A B|S-1| A B\rangle$. However we are not interested in the total cross-section but rather in the total hard cross-section with large momenta of observed partons. In the diagrammatic language this crosssection corresponds to cutting the forward scattering amplitude through the intermediate partonic propagators, with the restriction that the cut partons should have a large transverse momentum component.

One can standardly present all contributions to (5) - (7) in terms of diagrams which show partonic interactions via the amplitudes $i a_{i j}$. External partons correspond to those present in the colliding nuclei. They are assumed to have small transverse momenta and to be distributed in $x$ and $b$ according to $\left|\Psi_{A(B), n}\right|^{2}$. All diagrams can be separated into tree diagrams and diagrams with loops. In the tree diagrams all transferred momenta are small ("zero"), so that they are expressed through the forward scattering amplitudes $i a_{i j}$. Loop diagrams involve integrations over the intermediate parton momenta, which may be large. So they cannot be expressed via the forward scattering amplitudes $i a_{i j}$ but rather involve these amplitudes at large transferred momenta.

If one restricts oneself to the tree diagrams then the only way to find intermediate partons with high transverse momenta is to cut the forward scattering amplitudes $i a_{i j}$ converting them into the total (elastic) partonic cross-section $\sigma^{e l}$. According to the AGK rules, the sum over all possible cuts gives the final hard cross-section in the form $\langle A B|S-1| A B\rangle$ with $i a_{i j}$ substituted by $-\sigma_{i j}^{e l}$ in (7).

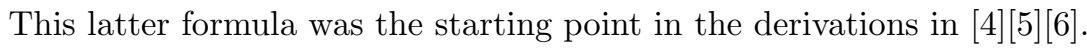

However loops give new contributions to the hard cross-sections, since cutting the loop produces intermediate partons with large transverse momenta. The result cannot be expressed in any simple way via the partonic cross-sections $\sigma^{e l}$, since it involves partonic amplitudes at large momentum transfers. Unfortunately it is easy to check that, at least perturbatively, the loop contributions are dominant. The reason is that the partonic amplitude $a$ is predominatly real (of order $\alpha_{s}$, whereas its imaginary part (and $\sigma^{e l}$ ) is of the order $\alpha_{s}^{2}$ and thus supressed by a factor $\alpha_{s}$ ). As an explicit example, let us compare the different contributions shown in Fig. 1. The external lines of the interacting partons supply a factor $A^{1 / 3}$ or $B^{1 / 3}$ each, due to the partonic densities in the nuclei. By cutting the single scattering diagram $a$ one obtains a contribution of order $(A B)^{1 / 3} \alpha_{s}^{2}$, while its iteration $b$ gives a contribution of order $(A B)^{2 / 3} \alpha_{s}^{4}$. The contributions of the cuts of the Glauber-like diagrams $c$ and $d$ are of orders $A^{1 / 3} B^{2 / 3} \alpha_{s}^{4}$ and $A^{2 / 3} B^{1 / 3} \alpha_{s}^{4}$. Tree diagrams with 3 interactions, as diagram $e$ in the figure, give a contribution of order $(A B)^{2 / 3} \alpha_{s}^{6}$. The leading contribution of the loop diagram $f$ is, on the contrary of order $(A B)^{2 / 3} \alpha^{4}$. Since all 4 elementary amplitudes can now be taken uncut (and real) in that case, this last contribution is by far dominant, as compared to all connected diagrams, and comparable to the iterated lowest order diagram. It can be shown that a similar argument holds for any number of interacting partons: the dominant contribution is given by iterations of the sum of the lowest order diagrams plus loops. All other diagrams are suppressed by a factor $\alpha_{s}^{2}$.

As a consequence by substituting $i a$ by $-\sigma^{e l}$, which is the approximation used in [4] 河 [6], one only selects a limited, sub-leading, set diagrams, the tree diagrams. Notice that the situation is quite different as compared to the case of the Glauber picture of $\mathrm{AB}$ scattering in terms of nucleons. In that case the amplitude is in fact mainly imaginary and, in addition, loops are likely to be suppressed by finite formation time arguments. The inclusive hard cross-section, obtained in [4] [5] [6] in the tree diagram approximation, needs therefore to be re-derived keeping the contribution of loops into account. 


\section{Jet spectrum: general}

To find the spectrum of emitted jets we have to fix the final state of one of the nuclei in a specific manner, in which one of the partons appears with the observed wave function $\psi_{\alpha}(z)$. The choice of the observed parton is irrelevant, since the total wave function of the nucleus is supposed to be symmetric in all the partons, so we choose it to be the first one. Note that choosing the observed parton to belong to nucleus A implies studying the spectrum in the direction of nucleus A and conversely for nucleus B. In the following we study the former case, so we take

$$
\Psi_{A^{\prime}, n}\left(z_{1}, z_{2}, \ldots z_{n}\right)=\sqrt{n} \psi\left(z_{1}\right)_{\alpha} \tilde{\Psi}_{A^{\prime}, n-1}\left(z_{2}, \ldots z_{n}\right)
$$

The second factor represents the state of the remaining $n-1$ partons, together with the observed one. The factor $\sqrt{n}$ is to adjust the normalizations of the symmetrized wave functions $\Psi_{n}$ and $\tilde{\Psi}_{n-1}$. Using (13) we get the probability to observe a parton with wave function $\psi$ at a given impact parameter $\beta$ in the nucleus A-nucleus B scattering as

$$
\begin{gathered}
\frac{d \sigma_{\alpha}}{d^{2} \beta}=\sum_{n l, n^{\prime}, l^{\prime}} \sqrt{n n^{\prime}} \sum_{A^{\prime}, B^{\prime}} \int d \tau_{A}^{\prime}\left(n^{\prime}\right) d \tau_{B}^{\prime}\left(l^{\prime}\right) d \tau_{A}(n) d \tau_{B}(l) \\
\Psi_{A, n^{\prime}}^{*}\left(z_{i}^{\prime}\right) \Psi_{B, l^{\prime}}^{*}\left(u_{i}^{\prime}\right)\left[S_{n^{\prime} l^{\prime}}^{*}\left(z_{i}^{\prime} \mid u_{j}^{\prime}\right)-1\right] \psi_{\alpha}\left(z_{1}^{\prime}\right) \tilde{\Psi}_{A^{\prime}, n^{\prime}-1}\left(z_{2}^{\prime}, . . z_{n}^{\prime}\right) \Psi_{B, l^{\prime}}\left(u_{j}^{\prime}\right) \\
\psi_{\alpha}^{*}\left(z_{1}\right) \tilde{\Psi}_{A, n}^{*}\left(z_{2}, \ldots z_{n}\right) \Psi_{B^{\prime}, l}^{*}\left(u_{j}\right)\left[S_{n l}\left(z_{i} \mid u_{j}\right)-1\right] \Psi_{A, n}\left(z_{i}\right) \Psi_{B, l}\left(u_{j}\right)
\end{gathered}
$$

The sum over all intermediate partonic states, with a given number of partons, is done by using closure:

$$
\sum_{A} \Psi_{A, n^{\prime}}\left(z_{1}^{\prime}, \ldots z_{n^{\prime}}^{\prime}\right) \Psi_{A, n}^{*}\left(z_{1}, \ldots z_{n}\right)=\delta_{n n^{\prime}} \prod_{i=1} \delta\left(z_{1}^{\prime}, . ., z_{n} \mid z_{1}, \ldots, z_{n}\right)
$$

where the $\delta$ function is a symmetrized product of $\delta^{3}\left(z_{i}^{\prime}-z_{i}\right)$. We obtain

$$
\begin{gathered}
\frac{d \sigma_{\alpha}}{d^{2} \beta}=\sum_{n l} n \int d z_{1} d z_{1}^{\prime} \psi_{\alpha}\left(z_{1}^{\prime}\right) \psi_{\alpha}^{*}\left(z_{1}\right) d \tau_{A}(n-1) d \tau_{B}(l) \Psi_{A, n}{ }^{*}\left(z_{1}^{\prime}, z_{2}, \ldots z_{n}\right) \Psi_{A, n}\left(z_{1}, z_{2}, \ldots z_{n}\right)\left|\Psi_{B, l}\left(u_{j}\right)\right|^{2} \\
{\left[S_{n l}^{*}\left(z_{1}^{\prime}, z_{2}, \ldots z_{n} \mid u_{1}, \ldots, u_{l}\right)-1\right]\left[S_{n l}\left(z_{1}, z_{2}, \ldots z_{n} \mid u_{1}, \ldots u_{l}\right)-1\right]}
\end{gathered}
$$

Notice that by summing over all possible observable states $\psi_{\alpha}$ one gets

$$
\sum_{\alpha} \psi_{\alpha}\left(z_{1}^{\prime}\right) \psi_{\alpha^{*}}\left(z_{1}\right)=\delta\left(z_{1}^{\prime} \mid z_{1}\right)
$$

which implies

$$
\begin{gathered}
\sum_{\alpha} \frac{d \sigma_{\alpha}}{d^{2} \beta}=\sum_{n l} n \int d \tau_{A}(n) d \tau_{B}(l)\left|\Psi_{A, n}\right|^{2}\left(z_{i}\right)\left|\Psi_{B, l}\left(u_{j}\right)\right|^{2} \\
{\left[S_{n l}^{*}\left(z_{1}, z_{2}, \ldots z_{n} \mid u_{1}, \ldots, u_{l}\right)-1\right]\left[S_{n l}\left(z_{1}, z_{2}, \ldots z_{n} \mid u_{1}, \ldots u_{l}\right)-1\right]} \\
=\sum_{n l} n \int d \tau_{A}(n) d \tau_{B}(l)\left|\Psi_{A, n}\right|^{2}\left(z_{i}\right)\left|\Psi_{B, l}\left(u_{j}\right)\right|^{2} \\
{\left[2-S_{n l}^{*}\left(z_{1}, z_{2}, \ldots z_{n} \mid u_{1}, \ldots, u_{l}\right)-S_{n l}\left(z_{1}, z_{2}, \ldots z_{n} \mid u_{1}, \ldots u_{l}\right)\right]=<n>\sigma_{A B}^{t o t}}
\end{gathered}
$$

The result is expected: the integrated inclusive cross-section gives the total cross-section multiplied by the average multiplicity, which in our case is obviously $\langle n\rangle$. Since $\langle n\rangle$ is supposed to grow

with $A$ only linearly (or even less rapidly, if the EMC effect is taken into account) the multiplicity does not grow faster then $A^{1}$. 


\section{Jet spectrum: non-trivial and geometric contributions}

The product of $S$ matrices in (16) generates 4 terms which we rearrange as

$$
\begin{gathered}
{\left[S_{n l}^{*}\left(z_{1}^{\prime}, z_{2}, \ldots z_{n} \mid u_{1}, \ldots, u_{l}\right) S_{n l}\left(z_{1}, z_{2}, \ldots z_{n} \mid u_{1}, \ldots u_{l}\right)-1\right]} \\
-\left[S_{n l}^{*}\left(z_{1}^{\prime}, z_{2}, \ldots z_{n} \mid u_{1}, \ldots, u_{l}\right)-1\right]-\left[S_{n l}\left(z_{1}, z_{2}, \ldots z_{n} \mid u_{1}, \ldots u_{l}\right)-1\right]
\end{gathered}
$$

It is instructive to study the meaning of the three terms from the point of view of the intermediate physical partonic states, corresponding to cutting the whole diagram with partonic interactions in the overall unitarity relation. In the second term only conjugate amplitudes $\left[i a_{i j}\right]^{*}$ enter, which means that this contribution corresponds to cut the interaction diagram from the extreme right, namely to the right of all partonic interactions. Conversely in the last term only the amplitudes $a_{i j}$ enter, which corresponds to cut the amplitude to the left of all partonic integractions. In both cases the momenta of the intermediate partons are convoluted with their initial distributions in the colliding nuclei, which is very narrow for geometrical reasons, so that also the resulting spectrum cannot be much broader.

To be more explicit, consider the third term in (18). Take the contribution from a single interaction of the first parton at impact parameter $b_{1}$ with some parton in B with its impact parameter $c$. Since we are interested in the distributions of partons from nucleus $\mathrm{A}$, as a function of the transverse momentum and at a fixed rapidity, we take

$$
\psi_{\alpha}\left(z_{1}\right)=e^{i p b_{1}} \delta\left(x_{1}-x\right)
$$

where $x$ and $p$ are the scaling variable and transverse momentum of the observed parton. Then the integration over $b$ and $c$ will take the form

$$
\int d^{2} b_{1} d^{2} c e^{i p b_{1}} \Psi_{A, n}\left(b_{1}, \ldots\right) i a\left(b_{1}-c\right)\left|\Psi_{B, n}(c, . .)\right|^{2}
$$

where we have suppressed all the variables, with the only exception of $b_{1}$ and $c$. The typical scale of

the partonic interaction is much smaller than the nuclear scale, so to a good approximation one can rewrite (20) as the product

$$
\int d^{2} b_{1} e^{i p b_{1}} \Psi_{A, n}\left(b_{1}, \ldots\right)\left|\Psi_{B, n}\left(b_{1}, . .\right)\right|^{2} \int d^{2} \operatorname{cia}(c)
$$

It is evident now that this expression is different from zero only for values of $p$ of the order of the inverse of the nuclear radius, unless one assumes that the nuclear distributions itself contains partons with high transverse momenta.

The non-trivial part of the partonic distribution originated by the hard collisions corresponds therefore to cutting the interaction diagram in between hard collisions and thus is totally contained in the first term in (18). Remarkably this contribution can be greatly simplified (differently with respect to the last two terms in (18)). In fact, using the factorization property (6) we can write

$$
S_{n l}\left(z_{1}, z_{2}, \ldots \mid u_{1}, \ldots u_{l}\right)=\prod_{j=1}^{l} s_{1 j} \prod_{i=2}^{n} \prod_{j=1}^{l} s_{i j}
$$

Due to the unitarity relation (8) the second product in (22) is cancelled by its conjugate in the product $S^{*} S$ in (18), so that we get

$$
S_{n l}^{*}\left(z_{1}^{\prime}, z_{2}, \ldots z_{n} \mid u_{1}, \ldots, u_{l}\right) S_{n l}\left(z_{1}, z_{2}, \ldots z_{n} \mid u_{1}, \ldots u_{l}\right)-1=\prod_{j=1}^{l}\left[1+i a\left(z_{1}^{\prime}, u_{j}\right)\right]^{*}\left[1+i a\left(z_{1}, u_{j}\right)\right]-1
$$


Putting this expression into (16) we note that (23) does not depend on the partonic variables $z_{2}, \ldots z_{n}$ of nucleus A. Integrating over $z_{2}, \ldots z_{n}$ and summing over $n$ one obtains the $\rho$ matrix of nucleus $\mathrm{A}$ as a function of the nuclear partonic degrees of freedom:

$$
\sum_{n} n \int d \tau_{A}(n-1) \Psi_{A, n}^{*}\left(z_{1}^{\prime}, z_{2}, \ldots z_{n}\right) \Psi_{A, n}\left(z_{1}, z_{2}, \ldots z_{n}\right)=\rho_{A}\left(z_{1} \mid z_{1}^{\prime}\right)
$$

This relation in fact defines the nuclear $\rho$-matrix. As a consistency check one may notice that, under the factorization assumption (11), at $z_{1}^{\prime}=z_{1},(24)$ reduces to $\Gamma\left(z_{1}\right)$.

The contribution from the 1st term in (18) may therefore be written as

$$
\frac{d \sigma_{\alpha}^{(1)}}{d^{2} \beta}=\sum_{l} \int d z_{1} d z_{1}^{\prime} \psi_{\alpha}\left(z_{1}^{\prime}\right) \psi_{\alpha}^{*}\left(z_{1}\right) \rho_{A}\left(z_{1} \mid z_{1}^{\prime}\right) d \tau_{B}(l)\left|\Psi_{B, l}\left(u_{j}\right)\right|^{2}\left\{\prod_{j=1}^{l}\left[1+i a\left(z_{1}^{\prime}, u_{j}\right)\right]^{*}\left[1+i a\left(z_{1}, u_{j}\right)\right]-1\right\}
$$

\section{The hard spectrum}

To obtain a more explicit form of the spectrum we make use of the factorization ansatz (11) for nucleus B:

$$
\left|\Psi_{B, l}\left(u_{j}\right)\right|^{2}=\frac{1}{l !} e^{-\langle l\rangle} \prod_{j=1}^{l} \Gamma\left(u_{i}\right)
$$

with

$$
\langle l\rangle=\int d^{3} u \Gamma_{B}(u)
$$

We also assume for the parton distributions the factorized expression

$$
\Gamma_{B}(u)=T_{B}(c) P_{B}(w)
$$

where $w$ and $c$ are the fractional momentum and transverse parton coordinate respectively. The normalizations are

$$
\int d^{2} b T_{B}(c)=1, \quad \int d w P_{B}(w)=\langle l\rangle
$$

Expressing the wave function of the observed parton as in Eq.(19), we obtain

$$
\begin{gathered}
(2 \pi)^{2} \frac{d \sigma^{(1)}}{d^{2} \beta d y d^{2} p}=\sum_{l} \frac{1}{l !} e^{-\langle l\rangle} \int d^{2} b_{1} d^{2} b_{1}^{\prime} e^{i p\left(b_{1}-b_{1}^{\prime}\right)} \rho_{A}\left(x, b_{1}-\beta \mid x, b_{1}^{\prime}-\beta\right) \\
\prod_{j=1}^{l} d^{2} c_{j} d w_{j} T_{B}\left(c_{j}\right) P_{B}\left(w_{j}\right)\left\{\prod_{j=1}^{l}\left[1+i a\left(z_{1}^{\prime}, u_{j}\right)\right]^{*}\left[1+i a\left(z_{1}, u_{j}\right)\right]-1\right\}
\end{gathered}
$$

with $z=(x, b)$ and $z^{\prime}=\left(x, b^{\prime}\right)$. Taking the origin in the center of nucleus $\mathrm{B}$, so that the partonic distribution in nucleus $\mathrm{A}$ becomes shifted by the overall impact parameter $\beta$, due to factorization, the integrations over $\left(w_{j}, c_{j}\right)$ give the $l$-th power of

$$
J=\int d^{2} c d w T_{B}(c) P_{B}(w)\left[1+i a\left(x, w ; b_{1}^{\prime}-c\right)\right]^{*}\left[1+i a\left(x, w, b_{1}-c\right)\right]
$$

where the dependence of the scattering amplitude on the transverse distance between the interacting partons is explicit. The different terms in this integral are treated as in the standard Glauber derivation. Let us consider the term with the product of two amplitudes. The distance between the interacting partons for hard interactions is very small as compared to the nuclear distances. So using 
$r=b_{1}-c$ as a integration variable we find $c=b_{1}-r$ and we can take the nuclear profile function out of the integral at $c \simeq b_{1}$. We obtain

$$
T_{B}\left(b_{1}\right) \int d^{2} r d w P_{B}(w) a^{*}\left(x, w ; b_{1}^{\prime}-b_{1}+r\right) a(x, w ; r) \equiv T_{B}\left(b_{1}\right) F_{B}\left(x, b_{1}^{\prime}-b\right)
$$

where

$$
F_{B}(x, b)=\int d^{2} r d w P_{B}(w) a^{*}(x, w ; b+r) a(x, w ; r)=\int d w P_{B}(w) \int \frac{d^{2} p}{(2 \pi)^{2}} I(p) e^{i p b}
$$

is the Fourier transform of the transverse momentum distribution in the parton-parton interaction, averaged over the parton rapidity distribution of nucleus B. Note that at $b=0$ (33) gives the total cross-section for parton-parton collisions (which coincides with the elastic cross-section), averaged over the rapidities of the B-partons

$$
\sigma_{B}^{t o t}(x)=\sigma_{B}^{e l}(x)=\int d w P_{B}(w) \int d^{2} r|a(x, w ; r)|^{2}
$$

The other terms in (31) are evaluated in a similar manner, the result is

$$
J=\langle l\rangle+T_{B}\left(b_{1}\right) F_{B}\left(x, b_{1}^{\prime}-b\right)-T_{B} F_{B}(x, 0)
$$

Taking the $l$-th power of (35) and summing over all values of $l$ one gets

$$
\exp \left(T_{B}\left(b_{1}\right)\left[F_{B}\left(x, b_{1}^{\prime}-b_{1}\right)-F_{B}(x, 0)\right]\right)
$$

The term in (30) with unity gives unity after summation over $l$, so that the cross section is expressed as

$$
\begin{gathered}
(2 \pi)^{2} \frac{d \sigma^{(1)}}{d^{2} \beta d y d^{2} p}=\int d^{2} b_{1} d^{2} b_{1}^{\prime} e^{i p\left(b_{1}-b_{1}^{\prime}\right)} \rho_{A}\left(x, b_{1}-\beta \mid x, b_{1}^{\prime}-\beta\right) \\
\left\{e^{T_{B}\left(b_{1}\right)\left[F_{B}\left(x, b_{1}^{\prime}-b_{1}\right)-F_{B}(x, 0)\right]}-1\right\}
\end{gathered}
$$

Introducing the integration variables $r=b_{1}^{\prime}-b_{1}$ and $b=(1 / 2)\left(b_{1}^{\prime}+b_{1}\right)$ and keeping into account that $r$ is a small quantity on the nuclear scale, one obtains

$$
(2 \pi)^{2} \frac{d \sigma}{d^{2} \beta d y d^{2} p}=\int d^{2} b d^{2} r e^{i p r} \rho_{A}(x, b-\beta)\left\{e^{T_{B}(b)\left[F_{B}(x, r)-F_{B}(x, 0)\right]}-1\right\}
$$

Here $\rho_{A}(x, b)=T_{A}(b) P_{A}(x)$ is just the partonic distribution of nucleus A. Since $F_{B}(x, r)$ goes to zero as $r \rightarrow \infty$ the inclusive cross-section (37) contains also a soft component of the spectrum, which in the expression is represented by the term proportional to $\delta^{2}(p)$. To remove it we subtract from the integrand its value at $r=\infty$. Our final expression for the inclusive cross-section is therefore

$$
(2 \pi)^{2} \frac{d \sigma}{d^{2} \beta d y d^{2} p}=\int d^{2} b d^{2} r e^{i p r} T_{A}(b-\beta) P_{A}(x)\left\{e^{T_{B}(b)\left[F_{B}(x, r)-F_{B}(x, 0)\right]}-e^{-T_{B}(b) F_{B}(x, 0)}\right\}
$$

The only trace in (38) of the nucleus $\mathrm{A}$ is in the term $T_{A}(, b-\beta) P_{A}(x)$, which appears as a weight factor for the different contributions from the various parts of nucleus B. For central collisions one can separate it as a nearly constant factor, so that the form of the spectrum should be practically independent of A and, in particular, it coincides with the spectrum in hadron-nucleus B collisions.

As mentioned in the Introduction, this formula coincides with the one obtained earlier in 国胞河] in the tree-diagram approximation. Remarkably, although dominant in the diagrammatic expansion of the forward amplitude, as a consequence of unitarity and of the cutting rules, there is no effect of loops to the inclusive cross section.

Notice also that, upon integration over all $p$, one obtains from (38) the total multiplicity of jets from nucleus A multiplied by the cross-section at a given impact parameter (the latter quantity being very close to unity for a heavy nucleus). The result coincides with the average number of wounded

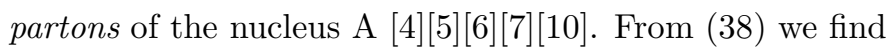

$$
\frac{d \sigma}{d^{2} \beta d y}=\int d^{2} b T_{A}(b-\beta) P_{A}(x)\left\{1-e^{-T_{B}(b) F_{B}(x, 0)}\right\}
$$




\section{Numerical results}

The distribution in rapidity and transverse momentum, Eq.(38), is obtained form the elementary inclusive cross-section $I(u, w, ; p)$ of two partons colliding with fractional momenta $u$ and $w$. For the gluon- gluon scattering one has [2] [3]

$$
\frac{d \sigma}{d y d^{2} p}=\frac{9 \alpha_{s}^{2}}{2 p^{4}} \int d y^{\prime} d u d w u G(u) w G(w)\left(1-\frac{p^{2}}{s u w}\right)^{3} \delta\left(u-x-\frac{p^{2}}{s x^{\prime}}\right) \delta\left(w-x^{\prime}-\frac{p^{2}}{s x}\right)
$$

with

$$
x=\frac{p}{\sqrt{s}} e^{y}, \quad x^{\prime}=\frac{p}{\sqrt{s}} e^{-y^{\prime}}
$$

the fractional momenta of the observed and recoiling gluons and $G(u), G(w)$ the distributions of the initial gluons, which we assume to have a small transverse momentum component. Eq. (40) exhibits a kinematical constraint between the longitudinal and transverse momenta of the final gluons. In particular their scaling variables are somewhat lower than those of the initial gluons. Rigorously speaking this goes beyond the Glauber approximation, which, as stated above, assumes the conservation of longitudinal momenta. At large $s$ however the problem is felt only close to the limiting value $p^{2} \sim p_{\max }^{2}=s u w / 4$. For $p$ much smaller than its limiting value, at $y^{\prime}<0$ one can take $G(u)$ out of the integral (40), at a value of momentum fraction corresponding to the momentum of the observed gluon, $u=x$. The rest of the integral can be transformed to the integration variable $w$. In the region $y^{\prime}>0$ one can similarly take $G(w)$ out of the integral and transform the integration to the variable $u$. In this way one obtains the final cross-section in a quasi-Glauber form (see Appendix 1 . for the details).

$$
\frac{d \sigma}{d y d^{2} p}=\frac{9 \alpha_{s}^{2}}{p^{4}}\left[x G(x) \int_{w_{\text {min }}} \frac{d w}{w} w G(w)\left(1-\frac{p^{2}}{s x w}\right)^{2}+(y \rightarrow-y)\right]
$$

where

$$
w_{\min }=\frac{p}{\sqrt{s}}\left(1+e^{-y}\right)
$$

We have checked that the approximations made in reducing (40) to (41) at energies above $200 \mathrm{GeV}$ change the spectrum by no more than $10 \%$ for $p<40 \mathrm{GeV} / \mathrm{c}$. The two terms in (41) have evidently the meaning of the contributions from the projectile and target partons. At $y=0$ they are naturally equal. To take into account the contribution of quarks, following [2] 3] [11], we introduce an effective parton density $P(x)$ and make the substitution

$$
x G(x) \rightarrow x P(x)=x G(x)+\frac{4}{9}[x Q(x)+x \bar{Q}(x)]
$$

Finally, to fit the existing experimental data, we multiply the cross-section by a $K$-factor. With $K=3$ and the partonic densities taken from [12] we obtain a rather satisfactory agreement with the experimental data on jet production on protons at $200 \mathrm{GeV}$ [13], as illustrated in Fig. 2.

The actual expression of the elementary inclusive cross section entering in Eq.(33) is

$$
I(x, w ; p)=(2 \pi)^{2} K \frac{9 \alpha_{s}^{2}}{p^{4}}\left(1-\frac{p^{2}}{s x w}\right)^{2} \theta\left(s x w-p^{2}\left(1+e^{y}\right)\right)
$$

Of course the expression holds only for sufficiently high values of the transferred momenta. So in the integral (33) we standardly restrict the integration to $p>p_{0}$ where $p_{0}$ is the infrared cutoff parameter. We neglect the change in the initial nuclear parton distributions as compared to the protonic ones (the EMC effect) and take $P_{A}(x)=A P(x)$ where $P(x)$ is the effective partonic distribution in the proton (42). In our calculations we use the partonic densities of 12 at the leading order. 
We restrict ourselves to the production of jets at center rapidity $y=0$. To simplify the calculations we also consider central collisions $(\beta=0)$ of identical nuclei and take the nuclei as spheres of radius $R_{A}$ with a constant density, so that

$$
T_{A}(b)=\frac{2}{V_{A}} \sqrt{R_{A}^{2}-b^{2}}
$$

Then in (38) the integration over $b$ can be done explicitly and we get

$$
(2 \pi)^{2} \frac{d \sigma}{d^{2} \beta d y d^{2} p}=2 x A P(x) \int d^{2} r e^{i p r}\left(Z(z)-Z\left(z_{0}\right)\right)
$$

where

$$
\begin{gathered}
Z(z)=e^{z}\left(\frac{6}{z^{3}}-\frac{6}{z^{2}}+\frac{3}{z}\right)-1-\frac{6}{z^{3}} \\
\left.z(r)=\frac{3 A}{2 \pi R_{A}^{2}}(f(r)-f(0)), \quad z_{0}=-\frac{3 A}{2 \pi R_{A}^{2}} f(0)\right)
\end{gathered}
$$

and

$$
f(r)=\frac{9}{2} K \int d^{2} p^{\prime} e^{i p^{\prime} r} \theta\left({p^{\prime}}^{2}-p_{0}^{2}\right) \int_{w_{\min }} \frac{d w}{w} w P\left(w,{p^{\prime}}^{2}\right) \frac{\alpha_{s}^{2}\left(p^{\prime 2}\right)}{{p^{\prime}}^{4}}\left(1-\frac{{p^{\prime}}^{2}}{s x w}\right)^{2}
$$

In (48) we have chosen the scales for $\alpha_{s}$ and $P(w)$ to be equal to the transverse momentum squared of the recoil parton (i.e. to the square of the transverse momentum transferred to the observed jet). The factor 2 in (45) takes into account jets produced by projectile and target partons. For the running coupling constant we have taken $\Lambda_{Q C D}=0.3 \mathrm{GeV} / \mathrm{c}$ with four flavours.

Some care is needed in determining the scale of the initial partonic distribution $P(x)$. It is related to the momentum transferred in the first collision. In the case of a single collision the scale is of order $p^{2}$, the transverse momentum squared of the observed jet. In case of multiple collisions the scale may however be substantially smaller. In our calculations we separated the single scattering contribution from the general expression (45), where we have taken $P(x)$ at the scale $p^{2}$. In the remaining part, corresponding to double and higher scatterings, we have taken $P(x)$ at the scale $p_{0}^{2}$.

Even with the above simplifications the calculation of the final inclusive distribution is far from trivial since it involves direct and inverse Bessel transforms. We were able to obtain more or less stable results only for values of $p$ not higher than $\sim 20 \mathrm{GeV} / \mathrm{c}$, where, fortunately, we expect hard rescatterings to be most relevant.

We have considered central $\mathrm{Pb}-\mathrm{Pb}$ collisons at 200 and $6000 \mathrm{GeV}$, corresponding to RHIC and LHC energies. We have taken $p_{0}=2 \mathrm{GeV} / \mathrm{c}$ in accordance with the analysis of particle production data [13]. As for the $\mathrm{K}$-factor we have considered values in the range $1 \div 3$. As mentioned, at 200 $\mathrm{GeV}$ the value $K=3$ is favoured by the proton data [12]. The analysis in [14 found that while $p_{0}$ does not practically change with energy, the K-factor clearly diminishes to values close to unity.

In Figs. 3 and 4 we present our results in the form of ratios of the distribution (45), with all rescatterings included, to the contribution of the single scattering only (optical approximation). The latter is obviously just the proton-proton distribution multiplied by a nuclear factor

$$
F_{A}=\frac{9 A^{2}}{8 \pi R_{A}^{2}}
$$

\section{Conclusions}

Given the very large energies in heavy ion collision at the LHC, a common expectation is that global features of the typical event will be within reach of a perturbative QCD approach. It should nevertheless be emphasized the such a possibility is far from trivial, the capability of making statements on 
properties of an average inelastic event implying qualitative improvements of present understanding of strong interaction dynamics. In a typical heavy ion collision event at the LHC several different mechanisms, originated by the complexity of the interactions states, may play important roles giving rise to a rather structured interaction, which needs to be brought to light to gain the capability of making quantitative statements.

While an exhaustive description of the whole interaction process, even if limited to the hard component only, is still out of reach, various features which are likely to play an important role are presently under extensive investigation in the literature 15 16] 17. 18. The topic which we have addressed in the present paper is that of multiparton interactions, where several partons in the initial state are linked in a hard collision process, which is a rather natural mechanism to consider in a very dense interacting system. One of the reasons of interest is that such a process is able to restore the local isotropy in transverse space of the black disk limit of the interaction, which is broken by a two parton initiated process at the lowest orders in $\alpha_{S}[10]$.

Previous attempts to face this issue were based on a purely probabilistic description of the hard component of the multiparton interaction, which has a solid support for disconnected multiparton processes, while it has not such a strong basis in the case of a connected multiparton collision. Within an incoherent approach, interference effects are in fact neglected altogether, which, in particular, implies disregarding all off-diagonal contributions, namely ignoring the well known problem of loops in nucleus-nucleus collisions, which give, on the contrary, the dominant contribution to the overall interaction amplitude, as discussed in the first part of this paper. To approach the problem we have studied the simplest case where only elastic partonic interactions are taken into account and the overall nucleus-nucleus $S$-matrix is factorized as a product of elementary parton-parton $S$-matrices. In our approach production processes at the partonic level are therefore completely disregarded. Since the complexity of the interaction is described by the Glauber prescription of factorization of the $S$-matrix, any amplitude for a $n$-parton interaction process is represented by a convolution of twopartons interaction amplitudes, so that the parton-nucleus amplitude is expressed by a series of on shell rescattering terms. Notice that, as in the case of the canonical Glauber approach to hadronnucleus collisions, this by no means implies a space-time ordering between different interactions. It only implies that a connected $n$-body interaction process is well approximated by a product of two-body interactions, which basically means that, in a dispersive representation of the projectileexchanged gluon amplitude, the pole contribution is dominant. As for the quantitative relevance of hard rescatterings, our numerical study confirms that, at transverse momenta of $\sim 20 \mathrm{GeV}$, the effects are large both at RHIC and LHC energies.

The feature which, in our opinion, makes this approach interesting, even if some non-secondary features, as energy loss, are not included in the picture of the interaction, is that it allows one to obtain, without approximations, the inclusive transverse spectrum of minijets, which is therefore an exact consequence of the $S$-matrix considered. This feature allows one to argue that the result might represent a good starting point for the ambitious program of accomplishing an exhaustive description of hard spectra in nucleus-nucleus collisions.

\section{Acknowledgment}

This work was partially supported by the RFFI Grant 01-02-17137 of Russia and Italian Ministry of University and of Scientific and Technological Researches (MURST) by the Grant COFIN2001. 


\section{Appendix 1. Derivation of (41)}

Let us split the integration over $y^{\prime}$ in (40) in two parts, $y^{\prime}<0$ and $y^{\prime}>0$. Consider the part with $y^{\prime}<0$. It corresponds to observed gluons coming from the projectile. Integrating over $u$ we get for this part

$$
\left(\frac{d \sigma}{d y d^{2} p}\right)_{y^{\prime}<0}=\frac{9 \alpha_{s}^{2}}{p^{4}} \int_{p / \sqrt{s}} \frac{d x^{\prime}}{x^{\prime}} \int d w u G(u) w G(w)\left(1-\frac{p^{2}}{s u w}\right)^{3} \delta\left(w-x^{\prime}-\frac{p^{2}}{s x}\right)
$$

with $u=x+p^{2} /\left(s x^{\prime}\right)$. Further integration over $x^{\prime}$ gives

$$
\left(\frac{d \sigma}{d y d^{2} p}\right)_{y^{\prime}<0}=\frac{9 \alpha_{s}^{2}}{p^{4}} \int_{w_{\text {min }}} \frac{d w}{w} u G(u) w G(w) \frac{w}{w-p^{2} /(s x)}\left(1-\frac{p^{2}}{s u w}\right)^{3}
$$

where now

$$
u=x\left(1+\frac{x p^{2}}{s w-p^{2}}\right)
$$

and $w_{\text {min }}$ is determined from the condition that $x^{\prime}>p / \sqrt{s}$ which gives

$$
w_{\text {min }}=\frac{p}{\sqrt{s}}+\frac{p^{2}}{x s}
$$

Now we make our crucial approximation to neglect the second term in (53) and put $u=x$. This leads directly to the first term in (41).

The contribution from the region $y^{\prime}>0$ is transformed in a similar manner. Now we integrate over $w$ to get

$$
\left(\frac{d \sigma}{d y d^{2} p}\right)_{y^{\prime}>0}=\frac{9 \alpha_{s}^{2}}{p^{4}} \int^{p / \sqrt{s}} \frac{d x^{\prime}}{x^{\prime}} \int d w u G(u) w G(w)\left(1-\frac{p^{2}}{s u w}\right)^{3} \delta\left(u-x-\frac{p^{2}}{s x^{\prime}}\right)
$$

with $w=x^{\prime}+p^{2} /(s x)$. Further integration over $x^{\prime}$ gives

$$
\left(\frac{d \sigma}{d y d^{2} p}\right)_{y^{\prime}<0}=\frac{9 \alpha_{s}^{2}}{p^{4}} \int_{u_{\min }} \frac{d u}{u} u G(u) w G(w) \frac{u}{u-x}\left(1-\frac{p^{2}}{s u w}\right) * * 3
$$

Here

$$
w=\frac{p^{2}}{s x}\left(1+\frac{x}{u-x}\right)
$$

and $u_{\text {min }}$ is determined from the condition $x^{\prime}<p / \sqrt{s}$ to be

$$
u_{\min }=x+\frac{p}{\sqrt{s}}
$$

Again our approximation consists in neglecting the second term in (56). After that we obtain the second term in (41).

\section{Appendix 2. Fits to the existing jet data [7]}

The fits have the form

$$
\frac{d \sigma}{d y d p}=\frac{A}{\left(p+p_{0}\right)^{a}}, \frac{\mu \mathrm{b}}{\mathrm{GeV} / \mathrm{c}}
$$

Here follows the table of the parameters with the corresponding values of $\chi^{2}$ 


\begin{tabular}{|l|r|r|r|}
\hline & $200 \mathrm{GeV}$ & $500 \mathrm{GeV}$ & $900 \mathrm{GeV}$ \\
\hline $\mathrm{A}$ & $(0.977 \pm 0.047) \mathrm{E}+10$ & $(1.00 \pm 0.97) \mathrm{E}+10$ & $(1.00 \pm 0.98) \mathrm{E}+10$ \\
$\mathrm{a}$ & $8.028 \pm 0.095$ & $7.119 \pm 0.033$ & $6.433 \pm 0.034$ \\
$p_{0}$ & $4.14 \pm 0.32$ & $5.83 \pm 0.22$ & $8.50 \pm 0.28$ \\
$\chi^{2}$ & 3.84 & 5.514 & 16.4 \\
\hline $\mathrm{A}$ & $(0.91 \pm 0.48) \mathrm{E}+8$ & $(0.175 \pm 0.045) \mathrm{E}+8$ & $(0.379 \pm 0.065) \mathrm{E}+7$ \\
$\mathrm{a}$ & $6.68 \pm 0.23$ & $5.383 \pm 0.096$ & $4.402 \pm 0.068$ \\
$p_{0}$ & 2.0 (fixed) & 2.0 (fixed) & 2.0 (fixed) \\
$\chi^{2}$ & 4.54 & 10.87 & 33.6 \\
\hline
\end{tabular}

\section{Appendix 3. Details of the numerical calculation}

Integration over the angles transforms (45) and (48) in

$$
\frac{d \sigma}{d^{2} \beta d y d^{2} p}=\frac{1}{\pi} x A P(x) \int r d r \mathrm{~J}_{0}(p r)\left(Z(z)-Z\left(z_{0}\right)\right)
$$

and

$$
f(r)=9 \pi K \int_{p_{0}} p^{\prime} d p^{\prime} \mathrm{J}_{0}\left(p^{\prime} r\right) \frac{\alpha_{s}^{2}\left(p^{\prime 2}\right)}{p^{\prime 4}} \int_{w_{\text {min }}} \frac{d w}{w} w P\left(w, p^{\prime 2}\right)\left(1-\frac{p^{\prime 2}}{s x w}\right)^{2}
$$

where according to (40) and (41) at $y=0 x=p / \sqrt{s}$ and

$$
w_{\min }=\frac{2{p^{\prime}}^{2}}{s x}=\frac{2{p^{\prime}}^{2}}{p \sqrt{s}}
$$

One observes that the two integrations in (60) over $w$ y $p^{\prime}$ do not decouple, which makes the calculation rather complicated.

\section{Figure captions}

1. Diagrams illustrating the interactions of two partons from the projectile with two partons from the target.

2. Jet distributions in $p \bar{p}$ collisions at $200 \mathrm{GeV}$ at center rapidity. The lower curve is a fit to the experimental data [7]. The upper curve shows predictions from Eq. (41) with $K=3.0$.

3. Ratios of the total distributions to the single scattering contributions (optical approximation) for central $\mathrm{Pb}-\mathrm{Pb}$ collisions at $200 \mathrm{GeV}$ and center rapidity calculated from(45) with $p_{0}=2 \mathrm{GeV} / \mathrm{c}$.

4. Ratios of the total distributions to the single scattering contributions (optical approximation) for central $\mathrm{Pb}-\mathrm{Pb}$ collisions at $6000 \mathrm{GeV}$ and center rapidity calculated from(45) at $y=0$ with $p_{0}=2$ $\mathrm{GeV} / \mathrm{c}$.

\section{References}

[1] T. Affolder et al. [CDF Collaboration], Phys. Rev. D 64, 032001 (2001) [Erratum-ibid. D 65, 039903 (2002)] arXiv:hep-ph/0102074.

[2] K. J. Eskola, K. Kajantie and J. Lindfors, Nucl. Phys. B 323, 37 (1989).

[3] K. J. Eskola, K. Kajantie and K. Tuominen, Nucl. Phys. A 700, 509 (2002) arXiv:hep$\mathrm{ph} / 0106330$.

[4] G. Calucci and D. Treleani, Phys. Rev. D 41, 3367 (1990). 
[5] G. Calucci and D. Treleani, Phys. Rev. D 44, 2746 (1991).

[6] A. Accardi and D. Treleani, Phys. Rev. D 64, 116004 (2001) arXiv:hep-ph/0106306.

[7] A. Bialas, M. Bleszynski and W. Czyz, Nucl. Phys. B 111, 461 (1976).

[8] C.Pajares and A.V.Ramallo, Phys. Rev. D 31, 1985 (1975)

[9] K. G. Boreskov and A. B. Kaidalov, Sov. J. Nucl. Phys. 48, 367 (1988) [Yad. Fiz. 48, 575 (1988)].

[10] A. Accardi and D. Treleani, Phys. Rev. D 63, 116002 (2001) arXiv:hep-ph/0009234.

[11] G. Pancheri and Y. N. Srivastava, Phys. Lett. B 182, 199 (1986).

[12] M. Gluck, E. Reya and A. Vogt, Eur. Phys. J. C 5, 461 (1998) arXiv:hep-ph/9806404.

[13] C. Albajar et al. [UA1 Collaboration], Jets," Nucl. Phys. B 309, 405 (1988).

[14] K. J. Eskola and H. Honkanen, arXiv:hep-ph/0205048.

[15] R. Baier, Y. L. Dokshitzer, A. H. Mueller and D. Schiff, Nucl. Phys. B 531, 403 (1998) arXiv:hep$\mathrm{ph} / 9804212$.

[16] U. A. Wiedemann, Nucl. Phys. B 588, 303 (2000) arXiv:hep-ph/0005129.

[17] U. A. Wiedemann, Nucl. Phys. A 690, 731 (2001) arXiv:hep-ph/0008241.

[18] M. Gyulassy, P. Levai and I. Vitev, Phys. Rev. Lett. 85, 5535 (2000) arXiv:nucl-th/0005032. 

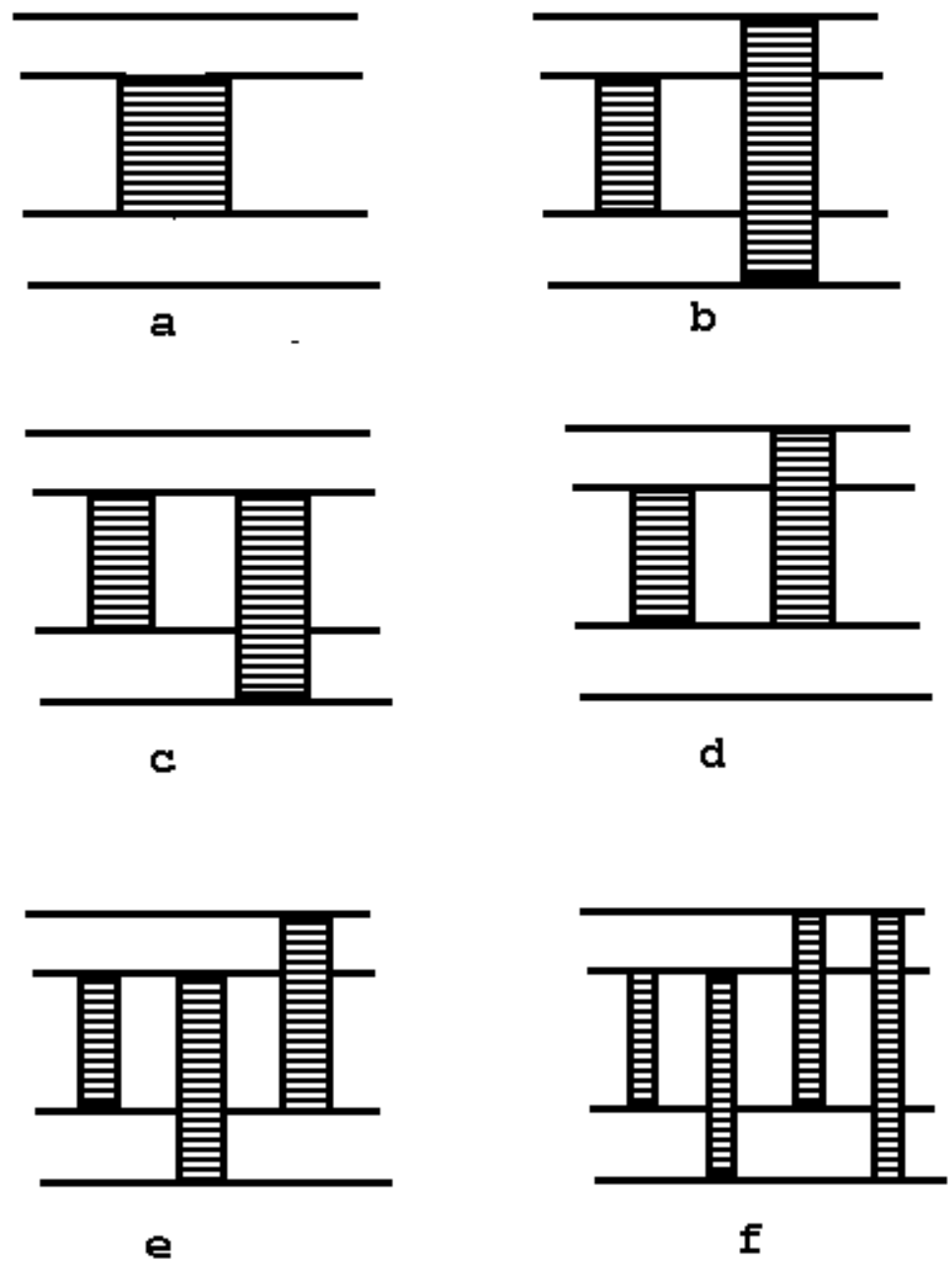


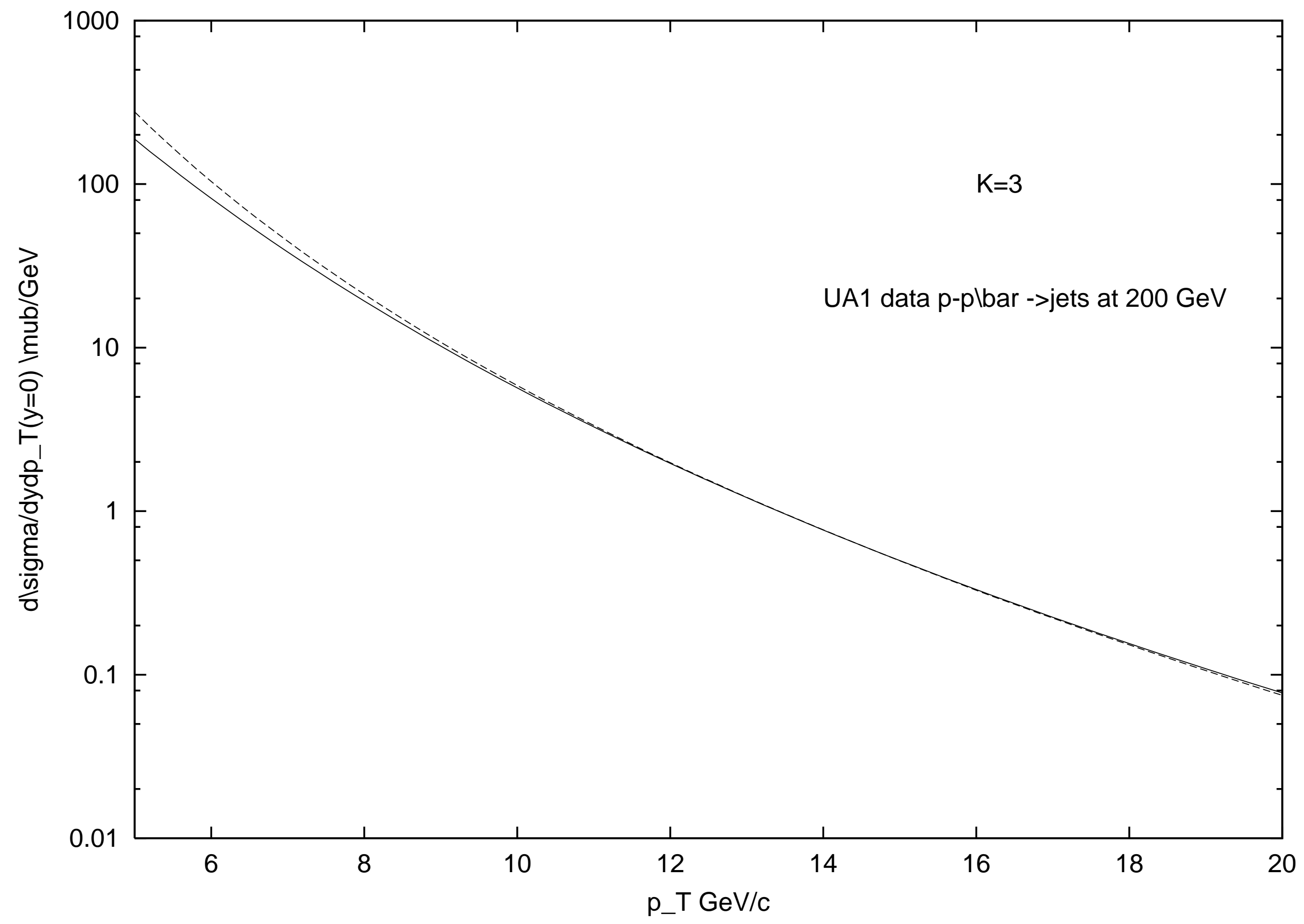




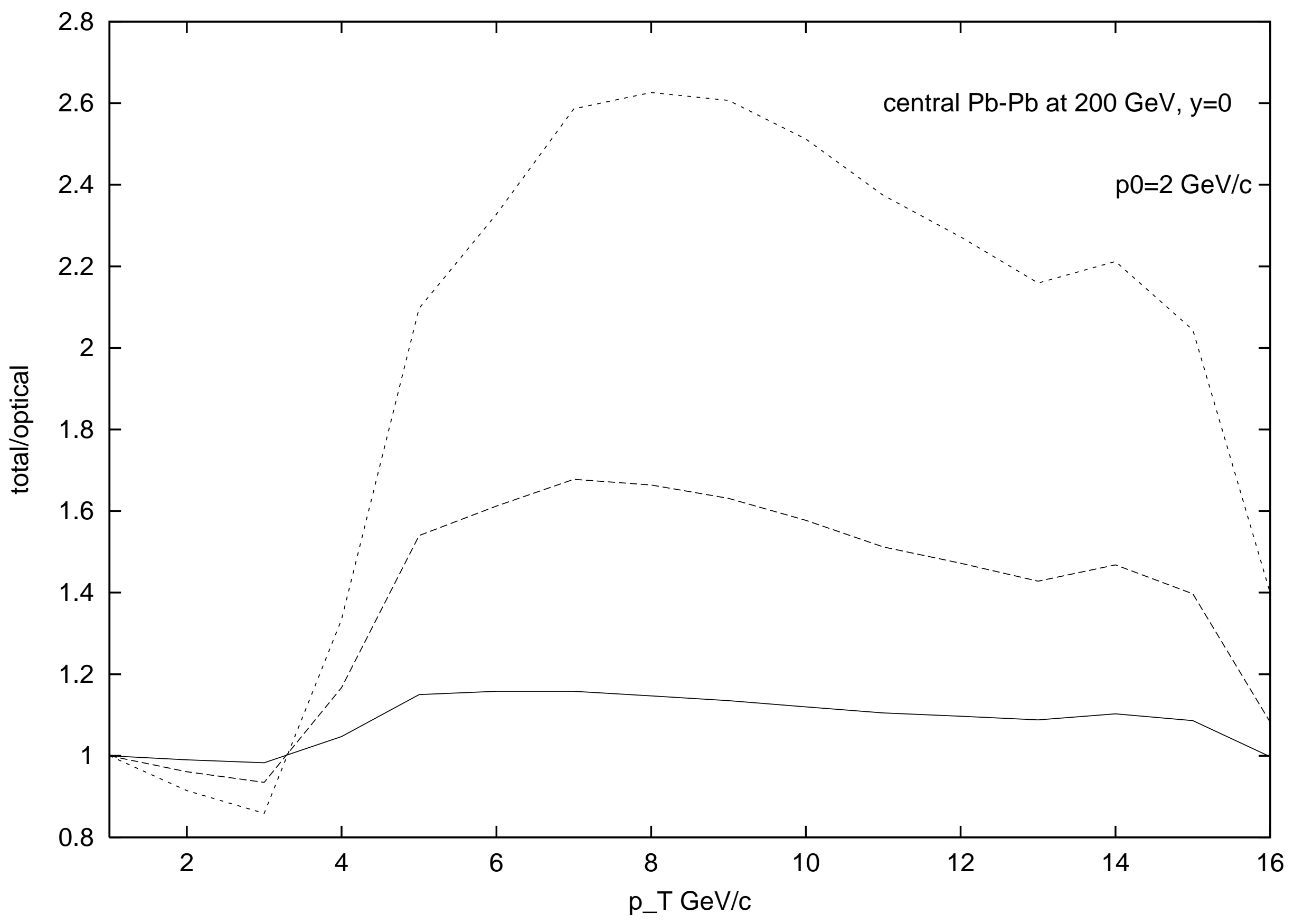




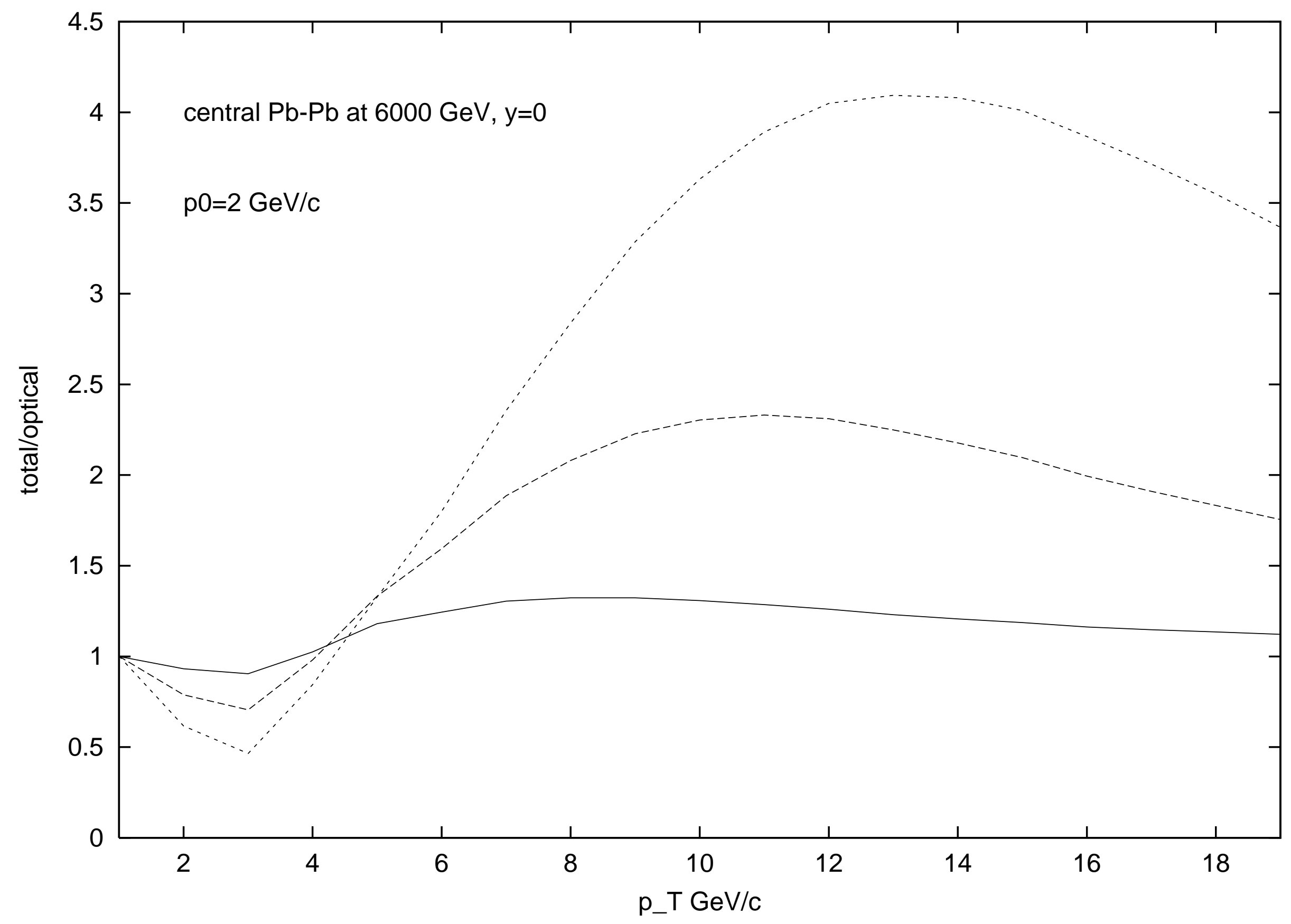

